\title{
Improvements in Recovery Efficiencies of Phenols from Phenol Fraction Using Two-Stage Alkali Treatment
}

\author{
G.V.S. Sarma ${ }^{1}$, P. Bala Bharathi ${ }^{1}$, J.V.S. Murty ${ }^{2}$, G.M.J. Raju ${ }^{1}$, K.V. Ramesh ${ }^{1 *}$, C. Bhaskara Sarma ${ }^{3}$ \\ ${ }^{1}$ Department of Chemical Engineering, Andhra University, Visakhapatnam-530003, India \\ ${ }^{2}$ Department of Chemical Engineering, GVPCOE(A), Visakhapatnam 530048, India \\ ${ }^{3}$ GVP College of Engineering for Women, Visakhaptnam 53048, India
}

Corresponding Author Email: kvramesh69@yahoo.com

https://doi.org/10.18280/ti-ijes.652-443

Received: 13 March 2021

Accepted: 29 May 2021

\section{Keywords:}

coke oven byproduct, dephenolized phenol, phenol fraction

\begin{abstract}
Experiments were carried out for the recovery of phenols from phenol fraction procured from tar distillation plant of Visakhapatnam steel plant by two stage alkali treatment, to study the effect of two-stage alkali treatment on the yield of phenols from phenol fraction. The results of the present investigation showed that two-stage alkali wash gives better yields of phenols compared to single stage alkali wash of the same phenol fraction with the same strength of alkali solution $(\mathrm{NaOH})$. Also it is shown that maximum yield of phenols could be obtained with $35 \%$ strength of alkali. In the first stage $70 \%$ of $35 \%$ strength solution was used while in the second stage treatment $30 \%$ of the same strength solution was used. Improvements in the recovery efficiencies of phenols were found to be $47 \%$ more than those reported earlier in the single-stage extraction studies for the same strength of alkali solutions.
\end{abstract}

\section{INTRODUCTION}

In the current scenario, efficient energy usage and recovery of minerals and useful substances from effluents are highly essential $[1,2]$. Hence, the present study is envisaged to obtain the augmentation levels of improvements in the yield of phenols from phenol fraction, a coke oven by-product using two-stage alkali treatment and optimizing the yield of phenols with respect to the concentration (strength) of the alkali and the ratios of alkali solution used in both the stages. In general, phenols are recovered from the phenol fraction obtained during the distillation of coal tar in steel plants. Phenols content of coal tar is $0.5-1.0 \%$. Phenol fraction contains around $18-22 \%$ phenol. To recover phenols from this fraction aqueous sodium hydroxide of strength around $10-12 \%$ is used in all commercial installations. It is now intended to find out the optimum strength of aqueous sodium hydroxide solution with respect to (i) the yields of regenerated phenols, (ii) the yields of de-phenolized oil, (iii) the effluent generation and (iv) loss of phenols in the effluent. The results of single stage extraction process using sodium hydroxide the strength of which was varied from $10-35 \%$ have been reported in literature [3].

The tar produced during high temperature carbonization of coal is a complex mixture of organic compounds containing essentially hydrocarbons. The quality of tar from the coke ovens is dependent upon the coking temperature, the temperatures in the ovens, free space temperatures in the ovens, conditions of the oven like flue gas leakages, undercharging, insufficient leveling of the charge after charging, aging of ovens, the hydraulic main pressure, etc. The tar is distilled in tar distillation plant into different fractions viz., light oil, phenol fraction, naphthalene fraction, wash oil, anthracene oil, and the residue after distillation yields pitch. The output of oils is about $35-45 \%$ of crude tar.

The phenol fraction boils between $180-210^{\circ} \mathrm{C}$, has a yield of $2-4 \%$ based on crude tar [4]. It contains $20-30 \%$ phenols, some naphthalene, pyridine bases and benzene homologues. The phenols are a mixture of phenols, cresols and xylenols. Phenols from phenol fraction are separated in the existing industrial practices, normally, by alkali treatment using $8-12 \%$ aqueous sodium hydroxide solution. Bhattacharya et al. [5] investigated the industrial applications of phenols and also reported in detail the factors augmenting the recovery of phenols from ammonical liquor, its effluents and from tar-oil cuts. The recovery of phenols from tar-oil cuts according to his investigations was found to be the best when $10 \%$ caustic soda was used for the separation of dephenolized phenol and water soluble phenolates. Investigations on Solvent extraction of phenols from coal tar fraction by Sen et al. [6] and recovery of phenols from the effluents of coal carburization processes by Chaudhuri et al. [7] using alkali treatment reported an optimum of $15 \% \mathrm{NaOH}$ concentration. Although some works [8] are reported on recovering phenol from waste water, to the best of the knowledge of the authors, no investigations on alkali treatment of byproduct phenolic fraction by furthering the concentration of caustic soda beyond $15 \%$ were reported in the literature.

Aruna Kumari [3] studied the extraction of phenols from phenol fraction containing $20-30 \%$ phenols using a single stage alkali wash (10-35\% concentration of sodium hydroxide solutions). When the phenol fraction is subjected to alkali wash, two immiscible layers namely aqueous layer and organic layer (unreacted phenol fraction) are formed. The sodium hydroxide in the alkali solution reacts with the phenols in the phenol fraction to form sodium phenoxide or sodium 
phenolate which is soluble in aqueous layer. The aqueous layer containing sodium phenolate is separated from the organic layer and treated with sulfuric acid to liberate phenols which form an organic layer and sodium sulfate the bi-product of the reaction forms the aqueous layer.

The stoichiometric chemical reactions for this process are:

(i) The reaction between phenols and alkali to form sodium phenolate is

$$
\mathrm{C}_{6} \mathrm{H}_{5} \mathrm{OH}+\mathrm{NaOH} \rightarrow \mathrm{C}_{6} \mathrm{H}_{5} \mathrm{ONa}+\mathrm{H}_{2} \mathrm{O}
$$

(ii) The regeneration reaction is

$2 \mathrm{C}_{6} \mathrm{H}_{5} \mathrm{ONa}+\mathrm{H}_{2} \mathrm{SO}_{4} \rightarrow 2 \mathrm{C}_{6} \mathrm{H}_{5} \mathrm{OH}+\mathrm{Na}_{2} \mathrm{SO}_{4}$

A two-stage extraction process has been carried out in the present study as it is expected to give better yields of regenerated phenols if the alkali of a given strength is added in two stages removing the products of reaction at each stage. Removing the reaction products formed during the first stage alkali treatment favors the forward reaction in the second stage increasing significantly the percentage of conversion of phenol fraction to phenol (Lechateliers Principle) thus augmenting the recovery efficiencies of regenerated phenols from phenol fraction. The study, hence, is aimed at increasing the yield of phenols by treating the phenol fraction on weight basis with six different concentrations of $\mathrm{NaOH}$ solution varied from $10 \%$ to $35 \%$ in two- stages. The experimental procedure for one experimental run has been described hereunder.

\section{EXPERIMENTAL PROCEDURE}

$\mathrm{NaOH}$ solution of known strength is prepared by accurately weighing the amounts of $\mathrm{NaOH}$ and dissolving it in required quantities of distilled water to obtain the desired strengths varying from $10 \%$ to $35 \%$. This solution is divided into two parts 50-50, 60-40, 70-30 as the case may be; the first part of the solution is used for extracting phenol during the first stage while the second part is used for the second final stage of extraction.

Fifty grams of phenol fraction is taken in a separating funnel, first part of $\mathrm{NaOH}$ solution of given strength (say $50 \mathrm{ml}$ in the first stage) is added and vigorously stirred for $1 / 2 \mathrm{hr}$ and the mixture is allowed to settle for about one hour when two layers are formed. The bottom layer is drained out into a beaker and kept aside for subsequent operation. The top layer is the organic layer containing Dephenolized Oil (DO). This is charged into another separating funnel and the second part of the $\mathrm{NaOH}$ solution (say the second $50 \mathrm{ml}$ of 50-50 ratio) is added and again vigorously stirred as done in the first stage and the solution is allowed to settle when again two layers are formed. The aqueous layer of the second stage is drained and now mixed with the aqueous layer obtained in the first stage kept aside in a beaker. The aqueous layers of both the stages are rich in sodium phenolate which is soluble in water. The top layer of the second stage (dephenolized oil) which is almost free of phenols is discarded.

The sodium phenolate solution in the beaker is now treated with $98 \%$ sulphuric acid for regenerating phenols. This is the regeneration step and this reaction is highly exothermic and therefore carried out slowly. On completion of the reaction, the regenerated phenol separates out as a top organic layer and bottom aqueous layer containing Sodium Sulphate solution. The reaction products of the organic layer (top layer) are analyzed for phenols and other components using a chromatographic analytical equipment of Schimadzu make. Similar procedure has been adopted for all the remaining strengths of alkali.

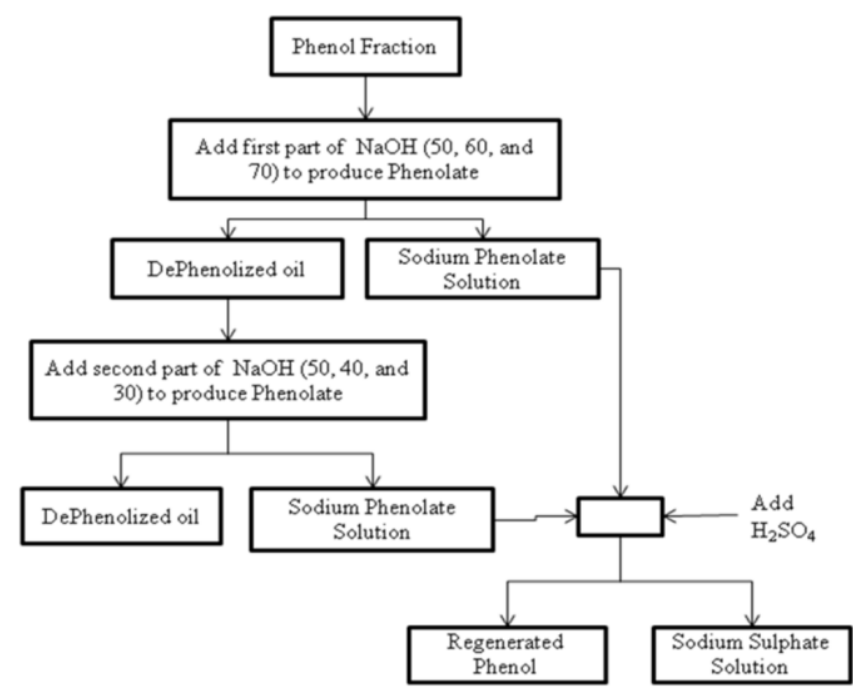

Figure 1. Procedure of two-stage alkali wash

Dehydration of regenerated phenols is carried out in a single stage distillation column by adding required quantities of toluene. The boiling point of phenol is $182^{\circ} \mathrm{C}$. Hence, the condensate was collected till the temperature rises to $110^{\circ} \mathrm{C}$ and slightly beyond this. The condensate contains water and toluene. These two are separated using separating funnel to recover toluene for subsequent dehydration operations. The residue, rich in Regenerated Phenol (RP) is almost completely free of moisture. The flow diagram for two-stage washing is shown below in Figure 1.

\section{RESULTS AND DISCUSSION}

The results obtained with double washing were compared with those of single washing using the same strengths of $\mathrm{NaOH}$ solutions. The measured experimental data were the weights of regenerated phenols, dephenolized oil and weights of the effluents. The calculated data were the percent weight losses.

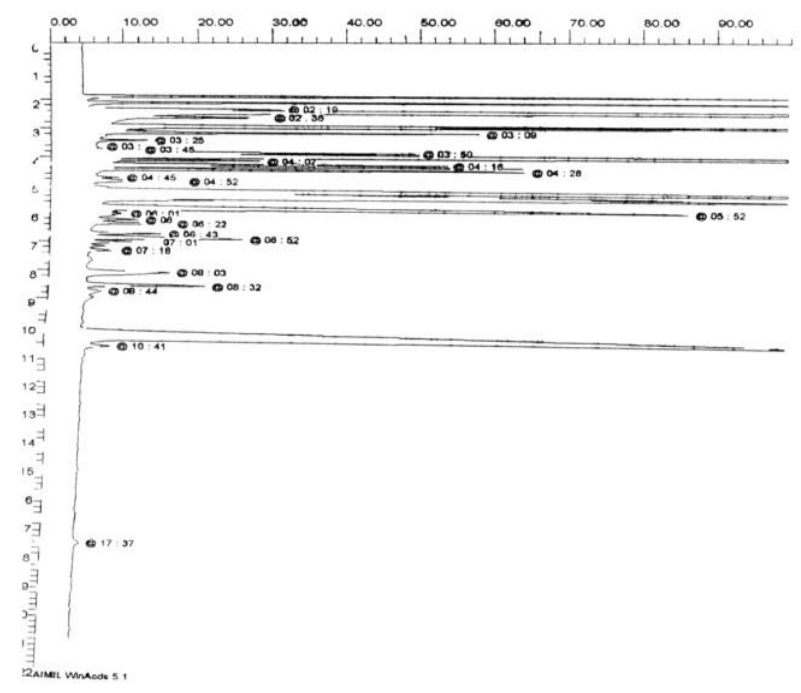

Figure 2. Phenol Fraction Gas Chromatogram (Phenol Fraction: Toluene $=1: 4)$. Sample Size: $0.5 \mu 1$, Column temperature: $100^{\circ} \mathrm{C}$ 
Earlier studies on extraction of phenols from phenol fraction employing single stage washing with the alkali by Aruna Kumari ${ }^{1}$ showed that the yield of crude phenol increased with increase in the strength of $\mathrm{NaOH}$. The gas chromatogram (shown in Figure 2) for phenol fraction procured from 'Visakhapatnam Steel Plant, Visakhapatnam' gives the analysis for its constituent components as: Phenol $11.2 \mathrm{~g}$.; ortho cresol $4.48 \mathrm{~g}$; Meta \& Para Cresol $6.05 \mathrm{~g}$; Naphthalene $14.24 \mathrm{~g}$ and xylenol.

\subsection{Yield of regenerated phenol (RP)}

The effect of alkali strength on the yield of phenols from phenol fraction is shown in Figure 3. For easy reference table 1 gives the experimentally measured and calculated data for two-stage alkali treatment. The data of earlier study on single stage alkali treatment is also shown for comparison in the same table. The data on weight of regenerated phenols (R P) expressed in $\mathrm{g} / 50 \mathrm{~g}$ of phenol fraction is shown plotted against alkali strength (\% of alkali) for all the three ratios of two-stage treatment, 50-50, 60-40 and 70-30. Yield of phenols has shown increasing trend with increase in $\mathrm{NaOH}$ concentration for all the three cases of washing indicating the improvements in recovery efficiencies. For the ratios 50/50 and $60 / 40$ the increase was found to be a maximum of $16 \%$, while for $70 / 30$ the increase with alkali concentration from 10 to $35 \%$ is drastic upto a maximum of $68 \% .50 \mathrm{~g}$ of phenol fraction yielded $22.92 \mathrm{~g}$ of phenols with $35 \% \mathrm{NaOH}(50 / 50)$, $23.24 \mathrm{~g}$ with $35 \% \mathrm{NaOH}(60 / 40)$ and $32.31 \mathrm{~g}$ with $35 \% \mathrm{NaOH}$ (70/30). 70/30 ratio for the two-stage treatment has shown improvements in the yield by $41 \%$ over that obtained with the ratio 50/50 for the same alkali strength i.e. $35 \%$. Two-stage washing for the same strength of alkali $(35 \%)$ showed a significant improvement in the yields of $\mathrm{R} P$ by $47 \%$ as against single stage washing of phenol fraction $(22.01 \mathrm{~g}$ of phenols in single stage washing).

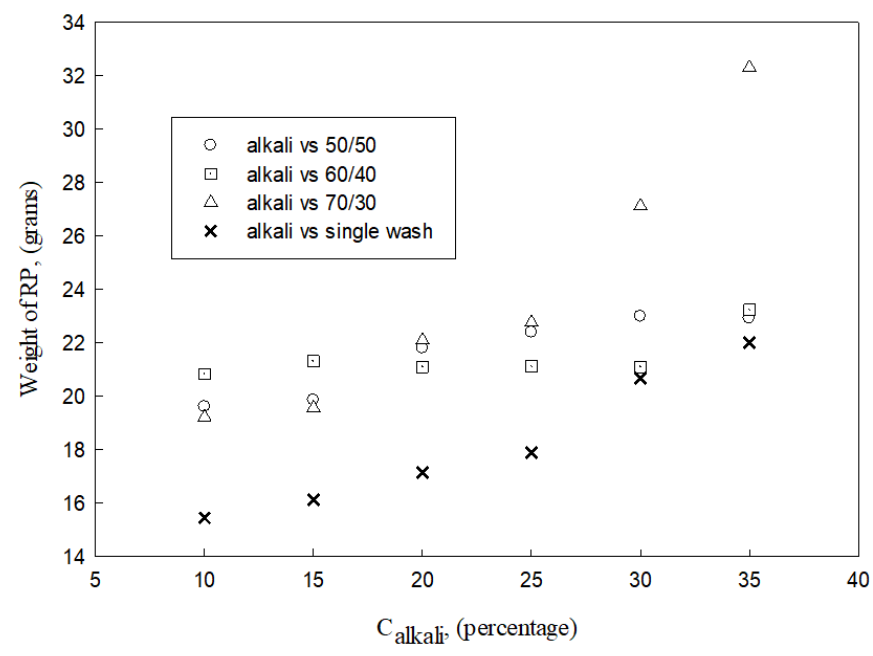

Figure 3. Effect of Alkali strength on the yield of Regenerated phenol (RP) in the aqueous layer

\subsection{Yield of dephenolized oil (DO)}

The extracted phenols as described earlier in sec .2.1 are removed as an aqueous solution as this dissolve in water and top layer; the organic layer is the dephenolized oil (D O) layer. Obviously, as expected the weight of the dephenolized oil layer decreases as the R P is increasing with the alkali strength.
The observations are shown in Figure 4 in which the weight data on Dephenolized oil is shown plotted against the alkali concentration. Even in this case a marginal decrease in the weight of D O was obtained for the cases of 50/50 and 60/40 ratios of $35 \% \mathrm{NaOH}$ strength while the fall in the weight of $\mathrm{D}$ $\mathrm{O}$ collected was very significant by $35 \%$ in case of $70 / 30$ ratio.

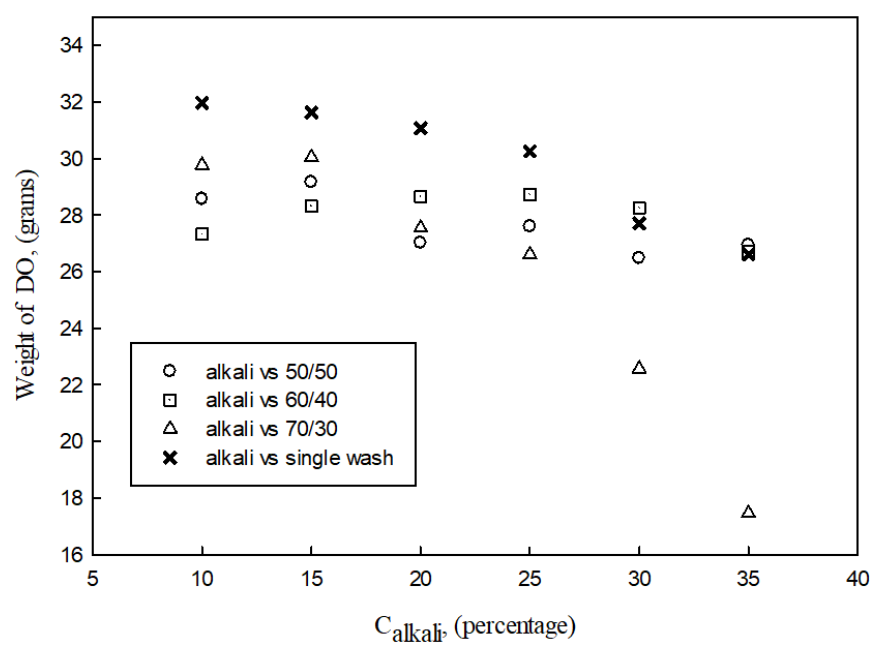

Figure 4. Effect of Alkali strength on the yield of dephenolized oil (DO) in the aqueous layer

\subsection{Losses in the experiment}

Calculated weight losses expressed in $\mathrm{g} / 50 \mathrm{~g}$ of phenol fraction are shown plotted in Figure 5. For all the strengths of Alkali losses showed a decreasing trend with increase in the strength of $\mathrm{NaOH} .10 \%$ strength alkali solution gave a maximum loss of $3.6 \mathrm{~g} / 50 \mathrm{~g}$ of raw phenol fraction while the losses were as low as $0.86 \mathrm{~g} / 50 \mathrm{~g}$ of phenol fraction for $35 \%$ strength solution. The losses were also found to be lower for the ratios 60/40 and 70/30 of alkali solution during two-stage treatment compared to those when 50/50 ratios were used. The decreasing trend in losses may be attributed to lesser effluent generation with increasing $\mathrm{NaOH}$ concentration. $10 \%$ strength of $\mathrm{NaOH}$ solution generated more effluent compared to $35 \%$ and so losses were found to be more at lower concentrations of $\mathrm{NaOH}$. For all the strengths of alkali, the losses in single stage alkali washings were found to be more by 2 to 4 times.

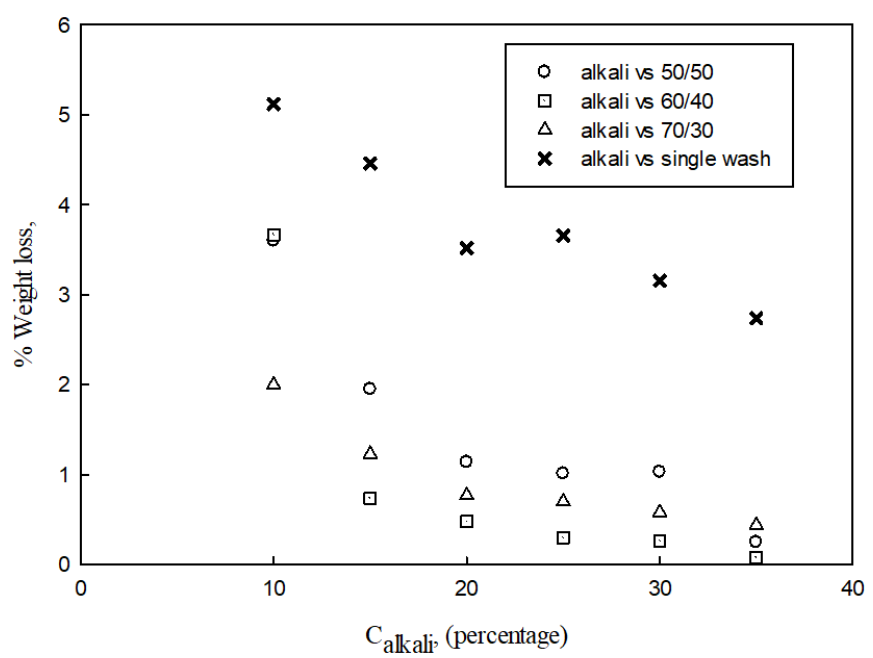

Figure 5. Effect of Alkali strength $\mathrm{NaOH}$ on \% Weight losses for both two-stage and single stage washing 


\subsection{Generation of effluent}

The two-stage alkali treatment as explained earlier involves three steps: The first step is the reaction between the phenol fraction and one part of sodium hydroxide solution of known strength. The top organic layer still contains some unconverted phenol fraction. So this is again treated with the remaining part of sodium hydroxide solution for complete conversion in the second step. In the third step the aqueous layer containing sodium phenolate in both the above steps is now treated with sulphuric acid when phenol is regenerated from sodium phenolate along with the other reaction product sodium sulphate. In the regeneration step, phenol forms the organic layer and sodium sulphate is in the aqueous layer. This aqueous layer separated from the organic layer forms the effluent. The effluent generation is thus due to the third regeneration step.

The amounts of effluents generated for all the strengths of alkali (10-35\%) used for washing or treatment is shown in the plots of Figure 6 . Generation of effluent is expected to be larger at low alkali concentrations due to larger amounts of water present. Weight data on effluent generated for all the strengths of alkali are plotted and shown in Figure 6. The fall in the effluent generation is drastic when the strength of alkali is increased. Similar trends were seen even in the case of single stage of treatment. For all the cases however, the trends in the fall of effluent generation were the same.

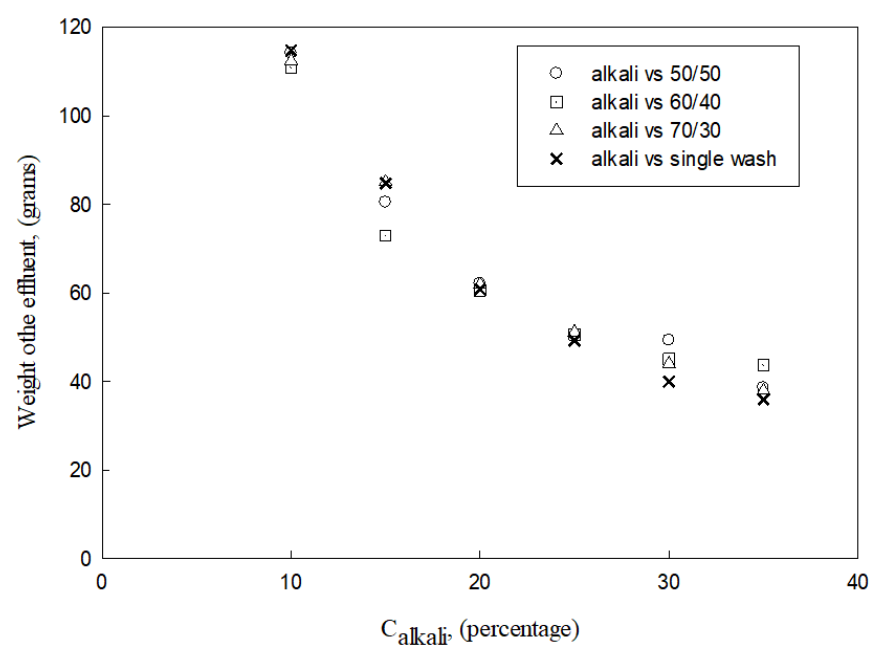

Figure 6. Effect of Alkali strength on effluent generation for both two-stage and single stage washing

\section{CONCLUSIONS}

The recovery efficiencies of regenerated phenols from phenol fraction and hence yields increased with increase in alkali strengths from 10-35 percent. Two-stage alkali treatment showed significant improvements in the yields and the improvements were 47 percent more than those obtained in single stage treatment. The 70-30 ratio of alkali solutions of any strength for first and second stage treatments gave maximum yields of phenols and the yield of dephenolized oil was greatly reduced. Losses were found to be lower in twostage treatment and were found to be minimum for an alkali strength of $35 \%$ at the treatment ratio of 70,30 for first and second stage treatments respectively. The lesser the strength of alkali the more is the effluent generation thus supporting the observations that higher strength of alkali will certainly augment the recovery efficiencies of phenols from phenol fraction.

\section{REFERENCES}

[1] Sreenivasulu, B., Ravali Sree, B., Srinivas, B., Ramesh, K.V. (2020). Heat transfer from two spheroids in tandem to a power law fluid by forced and mixed convection modes. Tecnica Italiana - Italian Journal of Engineering Science, 64: 331-340. https://doi.org/10.18280/tiijes.642-431

[2] Sarma, G.V.S., Raju, P.R.S., Ramesh, K.V., Raju, G.M.J. (2021). Recovery of manganese from ferromanganese slag by leaching with sulphuric acid. Tecnica Italiana Italian Journal of Engineering Science, 65: 33-35. https://doi.org/10.18280/ti-ijes.650105

[3] Aruna Kumari, S. (2005). Studies on Recovery Efficiencies of Crude Phenol from Phenol Fraction using Alkali Treatment, M.Tech dissertation. Andhra University, Visakhapatnam, India.

[4] Collin, G., Hoke, H. (1995). Ullmann's Encyclopedia of Industrial Chemistry, Vol. A26 (Wiley - VCH, Verlagsgesellschaft) Frankfurt/Main, Federal Republic of Germany.

[5] Bhattacharya, R.N., Roy, M.B., Tiwari, K.K. (1995). Technology Information Forecasting and Assessment council (TIFAC), Department of Science and Technology, New Delhi, India.

[6] Bhaduri, T.J., Sen, D.K., Tiwari, K.K., Nair, C.S.B. (1972). Proc. Symp. On chemicals and oil from coal, CFRI, Dhanbad, India. pp.51-88, 373-381.

[7] Chaudhuri, B., Patwardhan, A.A., Sharma, M.M. (1990). Alkylation of substituted phenols with olefins and separation of close-boiling phenolic substances via alkylation/dealkylation. Industrial \& Engineering Chemistry Research, 29(6): 1025-1031. https://doi.org/10.1021/ie00102a012

[8] Yang, X., Wang, B., Luo, H., Yan, S., Dai, J., Bai, Z. (2020). Efficient recovery of phenol from coal tar processing wastewater with TBP/DEC/cyclohexane: Extraction cycle and mechanism study. Chemical Engineering Research and Design, 157: 104-113. https://doi.org/10.1016/j.cherd.2020.03.005 